$$
\begin{array}{r}
\delta_{1} \omega=\omega^{\rho_{1}+1}, \text { and } \delta_{1} j=\omega^{\rho_{1}} v_{1} j+\omega^{\rho 2 v_{2}}+\cdots+\omega^{\rho_{z} v_{z}}<\omega^{\rho_{1}}\left(v_{1} j+1\right) \\
\sigma\left(\delta_{1} \mu, \delta_{1} j\right)<\sigma\left(\delta_{1} \mu, \omega^{\rho_{1}}\left(v_{1} j+1\right)\right)<\delta_{1} \mu+\omega^{\rho_{1}+1}=\delta_{1} \mu+\delta_{1} \omega .
\end{array}
$$

By $(2), \pi\left(\delta^{\mu}, \delta^{j}\right)<\omega^{\delta_{1} \mu+\delta_{1} \omega}=\left(\omega^{\delta_{1}}\right)^{(\mu+\omega)} \leqq \delta^{\mu+\omega} \leqq \delta^{\delta}$.

Hence by (1), the order type of $S$ is less than $\pi\left(\omega^{\delta}, \delta^{\delta}\right)$. This is a contradiction since $S$ was the segment of $M^{\delta}$ of order type $\pi\left(\omega^{\delta}, \delta^{\delta}\right)$.

UNIVERSITY OF ILLINOIS

\title{
A CHARACTERIZATION OF ABSOLUTE NEIGHBORHOOD RETRACTS
}

\section{RALPH H. FOX}

By an absolute neighborhood retract (ANR) I mean a separable metrizable space which is a neighborhood retract of every separable metrizable space which contains it and in which it is closed. This generalization of Borsuk's original definition ${ }^{1}$ was given by Kuratow$\mathrm{ski}^{2}$ for the purpose of enlarging the class of absolute neighborhood retracts to include certain spaces which are not compact. The space originally designated by Borsuk as absolute neighborhood retracts (or $\Re$-sets) will now be referred to as compact absolute neighborhood retracts. Many of the properties of compact ANR-sets hold equally for the more general ANR-sets. ${ }^{3}$

The Hilbert parallelotope $Q$, that is, the product of the closed unit interval $[0,1]$ with itself a countable number of times is a "universal" compact ANR in the sense that every compact ANR is homeomorphic to a neighborhood retract of $Q$. The classical theory of Borsuk makes good use of the imbedding of compact ANR-sets in $Q$. The problem solved here is that of finding a "universal" ANR.

Received by the editors June 28, 1941.

${ }^{1}$ Fundamenta Mathematicae, vol. 19 (1932), pp. 220-242.

${ }^{2}$ Fundamenta Mathematicae, vol. 24 (1935), p. 270, Footnote 1.

3 Ibid., pp. 272, 276, and 277, and Footnote 1, p. 279 and Footnote 3. Note that Theorem 12, Fundamenta Mathematicae, vol. 19 (1932), p. 229, is not true for general ANR-sets. In fact let $A=\sum S_{n}$ where $S_{n}$ is the plane circle of radius $2^{-n}$ and center $\left(3 \cdot 2^{-n}, 0\right)$; let $f(x, y)=(x,|y|)$ for $(x, y) \in A$ and let

$$
f_{n}(x, y)=\left\{\begin{array}{l}
(x,|y|), \text { for }(x, y) \in A-S_{n}, \\
(x, y), \quad \text { for }(x, y) \in S_{n} .
\end{array}\right.
$$

Then $f_{n} \rightarrow f$ in $A^{A} ; f$ can be extended to the half-plane $\{x>0\}$, but none of the maps $f_{n}$ can. $A$ is an ANR-set. Theorem 16, Fundamenta Mathematicae, vol. 19 (1932), p. 230, is also false for general ANR-sets.

${ }^{4}$ Fundamenta Mathematicae, vol. 19 (1932), p. 223. 
Strictly speaking, the problem as just stated has no solution; there is no single "universal" ANR, but rather a whole class of ANRsets which together serve in the "universal" capacity. Such a class of ANR-sets is the collection of subsets of the Hilbert parallelotope $Q \times[0,1]$ which contains the open $\operatorname{subset}^{5} Q \times(0,1]$ of $Q \times[0,1]$.

THEOREM 1. For a separable metrizable space $X$ the following three conditions are equivalent:

(1) $X$ is an ANR-set;

(2) There is a homeomorphism $f$ of $X$ into $Q$ such that $f(X) \times[0]$ is a neighborhood retract of $f(X) \times[0]+Q \times(0,1]$;

(3) $f(X) \times[0]$ is a neighborhood retract of $f(X) \times[0]+Q \times(0,1]$ for every homeomorphism $f$ of $X$ into $Q$.

$(1) \rightarrow(3)$ : If $f$ is a homeomorphism of an ANR-set $X$ into $Q$ then $f(X) \times[0]$ is an ANR-set. Since $Q$ is compact, so that

$$
\overline{f(X) \times[0]} \subset Q \times[0],
$$

it follows that $f(X) \times[0]$ is closed in $f(X) \times[0]+Q \times(0,1]$. Hence $f(X) \times[0]$ is a neighborhood retract of $f(X) \times[0]+Q \times(0,1]$.

$(3) \rightarrow(2)$ : Since $X$ is separable and metrizable a homeomorphism $f$ exists by Urysohn's theorem. ${ }^{6}$

$(2) \rightarrow(1)$ : Let $M$ be a separable metrizable space containing $X$ in which $X$ is closed and let $f$ be a homeomorphism of $X$ into $Q$. By Tietze's theorem ${ }^{7}$ there exists a continuous function $g$ defined on $M$ with values in $Q$ such that $g(x)=f(x)$ for every $x \in X$. Let $M$ be metrized, with metric $d$, and let $\rho(x)=\min \{1, d(x, X)\}$ for every $x \in M$. Let $h(x)=(g(x), \rho(x))$, so that $h$ is a continuous function defined on $M$ with values in $f(X) \times[0]+Q \times(0,1]$ which has the property $h(M-X) \subset Q \times(0,1]$. Let $V$ be a neighborhood of $f(X) \times[0]$ in $f(X) \times[0]+Q \times(0,1]$ and let $U=h^{-1}(V)$ so that $U$ is a neighborhood of $X$ in $M$. If $r$ is a retraction of $V$ onto $f(X) \times[0]$ then the mapping ${ }^{8}$ $f^{-1} \pi r h \mid U$, where $\pi$ denotes the projection of $Q \times[0]$ onto $Q$, is a retraction of $U$ onto $X$.

Kuratowski also gave an analogous generalization of the notion of absolute retract. ${ }^{2}$ According to the extended definition a separable metrizable space is an absolute retract (AR) if it is a retract of every containing separable metrizable space in which it is closed.

${ }_{5}^{5}$ The symbol $(0,1]$ denotes the half-open interval $0<t \leqq 1$.

${ }^{6}$ Alexandroff and Hopf, Topologie, p. 81.

7 Ibid., p. 73.

${ }^{8}$ If $B C B^{\prime}$ and $e$ is a function defined on $B^{\prime}$ then the notation $d=e \mid B$ means that $d$ is the function defined on $B$ such that $d(x)=e(x)$ for every $x \in B$. 
THEOREM 1'. For a separable metrizable space $X$ the following three conditions are equivalent:

(1') $X$ is an AR;

$\left(2^{\prime}\right)$ There is a homeomorphism $f$ of $X$ into $Q$ such that $f(X) \times[0]$ is a retract of $f(X) \times[0]+Q \times(0,1]$;

$\left(3^{\prime}\right) f(X) \times[0]$ is a retract of $f(X) \times[0]+Q \times(0,1]$ for every homeomorphism $f$ of $X$ into $Q$.

The proof of this theorem is an obvious modification of the preceding proof.

Corollary. If $C$ denotes the open $n$-cell $0<x_{i}<1(i=1, \cdots, n)$ and $D$ denotes the closed $n$-cell $0 \leqq x_{i} \leqq 1(i=1, \cdots, n)$ then any set $E$ such that $C \subset E \subset D$ is an AR.

By condition $\left(2^{\prime}\right)$ and a retraction of $Q \times[0,1]$ onto $D \times[0,1]$ it is sufficient to show that $E \times[0]$ is a retract of $E \times[0]+D \times(0,1]$. This can be done by projecting from the point $(1 / 2, \cdots, 1 / 2,-1)$ of Euclidean $(n+1)$-space.

It may be worth noting that conditions (2) and ( $\left.2^{\prime}\right)$ make possible a simpler proof of the Borsuk-Kuratowski ${ }^{9}$ theorem(s):

If $W$ is a closed subset of a normal space $Z$ and $X$ is an AR-set (ANR-set) then every continuous map of $W$ into $X$ can be extended to $Z$ (to a neighborhood of $W$ in $Z$ ).

In fact conditions (2) and $\left(2^{\prime}\right)$ replace a theorem of Kuratowski ${ }^{10}$ which involves infinite polyhedra.

Theorem 2. An ANR is locally contractible. ${ }^{11} A n \mathrm{AR}$ is also contractible.

Using (2) we can suppose that our ANR-set $Y$ is contained in $Q \times[0]$ and that there is a retraction $r$ of an open neighborhood $V$ of $Y$ in $Y+Q \times(0,1]$ onto $Y$. But $V$ is the intersection of $Y+Q \times(0,1]$ with an open set $V^{\prime}$ of $Q \times[0,1]$. Let $y \in Y$ and let $S_{\epsilon}$ denote the $\epsilon$-sphere in $Q \times[0,1]$ about the point $y$. Since $r$ is continuous there is a $\delta>0$ such that the intersection $T_{\delta}$ of the $\delta$-sphere $S_{\delta}$ and $Y+Q \times(0,1]$ is contained in $V^{\prime}$, hence in $V$, and $r\left(T_{\delta}\right) \subset S_{\epsilon}$. Let $u_{t}$ denote a contraction of $S_{\delta}$ to a point $p \in S_{\delta} \cdot(Q \times(0,1])$ which moves points rectilinearly, so that $u_{t}(x) \in Q \times(0,1]$ for every $0<t \leqq 1$ and

${ }^{9}$ Fundamenta Mathematicae, vol. 24 (1935), p. 275.

${ }^{10}$ Fundamenta Mathematicae, vol. 24 (1935), p. 266, Theorem 2.

${ }^{11}$ But not uniformly. See the example in Footnote 3. This theorem was proved by Borsuk, Fundamenta Mathematicae, vol. 19 (1932), p. 237 for compact ANR-sets. 
$y \in Y \cdot S_{\delta}$. Then $r u_{t} \mid Y \cdot S_{\delta}$ contracts $Y \cdot S_{\delta}$ in $Y \cdot S_{\epsilon}$. The second statement is a consequence of Theorem $3^{\prime}$.

THEOREM 3. A separable metrizable space $X$ is an ANR if and only if for every separable metrizable space $M$ containing $X$ (in whrch $X$ need not be closed!) there is a neighborhood $U$ of $X$ and a continuous function $h$ defined on $X \times[0]+U \times(0,1]$ with values in $X$ such that ${ }^{8}$ $h \mid X \times[0,1]$ is a deformation. ${ }^{12}$

Suppose $X$ is an ANR and $M$ a separable metrizable space containing $X$. We may assume that $M \subset Q$. By (2) and (3) there is an open neighborhood $V^{\prime}$ of $X \times[0]+Q \times(0,1]$ and a retraction $r$ of $V=V^{\prime} \cdot(X \times[0]+Q \times(0,1])$ onto $X \times[0]$. Let $\lambda(x)=d(x \times[0]$, $\left.Q \times[0,1]-V^{\prime}\right)$ for every $x \in M$ and let $U=\pi\left(V^{\prime} \cdot(Q \times[0])\right)$ where, as before, $\pi$ denotes the projection of $Q \times[0]$ onto $Q$. Define for every $(x, t) \in X \times[0]+U \times(0,1]$,

$$
\begin{aligned}
h(x, t) & =\pi r(x, t), & & \text { when } t \leqq \lambda(x), \\
& =\pi r(x, \lambda(x)), & & \text { when } t \geqq \lambda(x) .
\end{aligned}
$$

Since $\lambda$ is continuous and $\lambda(x)>0$ when $x \in U$ it follows that $h$ is continuous.

Conversely, let $U$ be a neighborhood of $X$ in $M=Q$ and let $h$ be a continuous function defined on $X \times[0]+U \times(0,1]$ with values in $X$ such that $h(x, 0)=x$ for every $x \in X$. Then $h$ is a retraction of $X \times[0]+U \times(0,1]$ onto $X \times[0]$. Furthermore $X \times[0]+U \times(0,1]$ is a neighborhood of $X \times[0]$ in $X \times[0]+Q \times(0,1]$.

Theorem 3'. A separable metrizable space $X$ is an $\mathrm{AR}$ if and only if for any separable metrizable space $M$ containing $X$ there is a continuous function $h$ defined on $X \times[0]+M \times(0,1]$ with values in $X$ such that ${ }^{8} h \mid X \times[0,1]$ is a contraction. ${ }^{12}$

Let $X$ be an AR and $M$ a separable metrizable space containing $X$; we may assume that $M \subset Q$. Let $r$ be a retraction of $X \times[0]+Q$ $\times(0,1]$ onto $X \times[0]$. Let $p \in Q$ and let

$$
h(x, t)=\pi r(t p+(1-t) x, t)
$$

for every $(x, t) \in X \times[0]+M \times(0,1]$, where $\pi$ is the projection of $Q \times[0]$ onto $Q$. Then $h$ maps $X \times[0]+M \times(0,1]$ continuously into $X$ and $h \mid X \times[0,1]$ is a contraction of $X$.

The converse is proved as in Theorem 3 .

${ }^{12}$ A deformation of $X$ is a continuous mapping $h$ of $X \times[0,1]$ into $X$ such that $h(x, 0)=x$ for every $x \in X$. If $h(X, 1)$ is a point then $h$ is called a contraction of $X$. 
If $X$ is locally compact the deformation $h \mid X \times[0,1]$ of Theorems 3 and $3^{\prime}$ can be chosen in advance of $M$. For then there exists ${ }^{13}$ a compact set $M^{*}$ and a homeomorphism $g$ of $X$ into $M^{*}$ such that $M^{*}-g(X)$ is a point. (We can suppose $X$ not compact so that $M^{*} \neq g(X)$.) Let $M^{*} \subset Q$. The homeomorphism $g$ can be extended ${ }^{13}$ to a continuous mapping $g^{*}$ of $\bar{X}$ into $M^{*}$ by defining $g^{*}(\bar{X}-X)=M^{*}$ $-g(X)$. The mapping $g^{*}$ of $\bar{X}$ into $Q$ can be extended, by Tietze's theorem, to a mapping $k$ of $M$ into $Q$. In the case of Theorem 3 let $h$ be the mapping of $X \times[0]+U \times(0,1]$ into $X$ defined by

$$
h(x, t)=g^{-1} \pi r(k(x), \min \{t, \lambda(x)\}),
$$

where $U=g^{-1} \pi\left(V^{\prime} \cdot(Q \times[0])\right)$. In the case of Theorem $3^{\prime}$ let $h$ be the mapping of $X \times[0]+M \times(0,1]$ into $X$ defined by

$$
h(x, t)=g^{-1} \pi r(t p+(1-t) k(x), t) .
$$

In both cases $h \mid X \times[0,1]$ is independent of $M$.

If $X$ is not locally compact it may not be possible to pick a deformation $h \mid X \times[0,1]$ satisfying the conditions of Theorems 3 or $3^{\prime}$ for all $M$. An example is the AR-set $\{0 \leqq x \leqq 1 ; y=0\}+\sum_{n=1}^{\infty}\{x=1 / n$; $0 \leqq y \leqq 1\}$.

\section{UNIVERSITY OF ILLINOIS}

${ }^{13}$ Alexandroff and Hopf, Topologie, I, p. 93. 